\title{
RF PHOTONICS: STATUS, CHALLENGES AND OPPORTUNITIES
}

\author{
Stephen A. Pappert \\ Electronics, Sensors, and Networks Research Division, Office of Naval Research, Arlington, VA

\section{Introduction}

Owing to inherently large time-bandwidth products and attractive size and weight features, photonics technology has long demonstrated utility for extending sensing and signal processing performance and capabilities. A few selected RF system areas where the broadband features of photonics are highlighted include RF signal generation/distribution, RF filtering/channelization, and A/D conversion. As commercial and military RF systems continue migrating to higher operating frequencies, the potential for increased bandwidth signal transmission and processing with high dynamic range using RF photonic technology continues to be an attractive option. This paper reviews the status of photonics relevant to RF signal transmission and processing and considers the serious challenge of high speed electronics. Opportunities for photonics will be assessed in light of competing technologies and trends for future RF systems.

\section{RF Photonics Link Technologies}

The performance of the analog optical link is critical for photonics to provide utility to RF systems. Efficient electrical-to-optical (E/O) conversion, optical-to-electrical $(\mathrm{O} / \mathrm{E})$ conversion, and low loss optical interconnects are imperative for photonic solutions to be competitive with conventional RF electronics. Optical fiber, with virtually lossless signal transport over frequencies and distances encountered in RF front-ends, provides an excellent starting point. $\mathrm{E} / \mathrm{O}$ and $\mathrm{O} / \mathrm{E}$ conversion losses coupled with high insertion loss integrated optics have traditionally impaired short-haul RF link performance. Recent progress in laser, modulator and detector technologies are beginning to remove this impairment and create new photonics insertion opportunities. An example of state-of-the-art performance for a short amplifierless photonic link include a noise figure of $<7.5$ $\mathrm{dB}$ across the full 1-12 $\mathrm{GHz}$ range, and $3.4 \mathrm{~dB}$ at $2 \mathrm{GHz}$ [1]. A high power, low noise laser, a high efficiency $\mathrm{LiNbO}_{3}$ integrated optical modulator, and a high power photodetector are keys to these results. As even lower noise figure photonic links are developed with improved $\mathrm{E} / \mathrm{O}$ and $\mathrm{O} / \mathrm{E}$ conversion devices, the need for any electronic amplification for receive-mode antenna operation can be eliminated. The spurious-free dynamic range (SFDR) is equally important and photonic links with SFDR $>125 \mathrm{~dB}-\mathrm{Hz}^{2 / 3}$ have been demonstrated [2]. These hero link results are indeed promising, but further emphasis is required in compact ruggedized packaging and link cost reduction for widespread deployment of microwave photonics into RF systems to occur.

\section{RF Photonic Signal Processing Technologies}

Moving beyond the functionality of RF transmission and signal distribution, a few highlighted areas of current RF Photonics interest include RF signal generation, RF filtering/channelization, and A/D converter signal pre-processing. High performance oscillators are critical in many RF system applications. OptoElectronic Oscillators (OEOs) are in development that uses high-Q optical storage elements to enable high quality microwave signal generation [3]. Present phase noise performance of an X-band OEO is comparable to the highest performing electronic oscillators at this frequency, with a significant size and weight advantage. In addition to pristine phase noise levels, experiments have demonstrated wideband OEO tunability over a decade of RF frequency with microsecond tuning speeds. The phase noise for this tunable OEO compares favorably to that of a "low phase noise" synthesizer based on tunable YIG technology.

RF signal pre-processing, filtering and channelization are additional technology areas where photonics can bring value to RF systems. As operational and instantaneous bandwidths continue to increase, RF front-end signal conditioning becomes critically important in preserving performance. Fixed and agile RF filters are good examples where photonic solutions can be unique. High-Q optical $\mu$-resonators and ring resonators for filtering applications have been demonstrated in various material systems and platforms. The highest fixed frequency single-pole filter optical $\mathrm{Q}$ has been demonstrated in a calcium fluoride $\left(\mathrm{CaF}_{2}\right) \mu$-sphere where an optical Q of $>10^{10}\left(<10 \mathrm{kHz}\right.$ linewidth) has been demonstrated [4]. For agile RF filtering, a 3-pole $\mathrm{LiNbO}_{3}$ tunable bandpass $\mu$-resonator filter has been demonstrated [5] with an insertion loss of $6 \mathrm{~dB}$, a $3 \mathrm{~dB}$ bandwidth of $30 \mathrm{MHz}$, an outof-band rejection of $>50 \mathrm{~dB}$, and is tunable in sub-microseconds over a $12 \mathrm{GHz}$ bandwidth. Compact size 
channelizers are also of interest in wideband RF systems and recent work based on parametric multicasting and spectral slicing has demonstrated [6] the ability to channelize an $18 \mathrm{GHz}$ instantaneous bandwidth with 250 $\mathrm{MHz}$ resolution bandwidth. Finally, high resolution wideband $\mathrm{A} / \mathrm{D}$ conversion is a persistent need for RF receivers and photonics has shown the ability to pre-process RF signals to extend the performance of electronic A/D converters. Recent performance levels that have been achieved include a 7 ENOB (Effective Number of Bits), $10 \mathrm{GHz}$ analog bandwidth demonstration that is readily scalable to higher performance and continuoustime operation [7]. This intermediate result is competitive with existing electronic quantizers.

\section{Electronics Challenge and Areas of Further Development}

The fundamental speed of semiconductor transistors (e.g. Si, SiGe, III-V) has advanced dramatically over the past few decades with the fastest electronic device technologies now approaching THz values for $\mathrm{f}_{\mathrm{T}}$ and $\mathrm{f}_{\max }$. These electronic advances can pose a serious threat to the use of RF photonics for high frequency and wide bandwidth applications. Solid-state electronics is now providing high performance wideband frequency coverage for antenna based systems to beyond $100 \mathrm{GHz}$. Mixed signal electronic circuit performance is also advancing as smaller and faster transistor technologies become available. It is safe to assume that electronics technology will continue to advance as more high frequency commercial and military applications emerge, further burdening RF photonics to show value and continue to improve performance.

The utility of photonics for RF systems improves as better analog transmission technology is developed. The photonic link is key and further cost and performance improvements are necessary for widespread deployment. Achieving low noise figure links at frequencies to $100 \mathrm{GHz}$ and beyond is highly desirable, and efficient broadband modulation and demodulation is clearly a high impact and challenging area of further development. A compact, high power, low noise $1.55 \mu \mathrm{m}$ laser is also an important area of further photonic device development. Shot-noise-limited laser performance over the entire microwave spectrum of interest with high wall plug efficiency is desirable. Improved link dynamic range is equally important. Broadband photonic link linearizers are needed to push the SFDR over the $130 \mathrm{~dB}-\mathrm{Hz}^{2 / 3}$ level. With this final ingredient, link gain, noise figure, dynamic range and bandwidth can be simultaneously achieved. These improvements are needed to help further the case for distributed analog photonic antenna interfacing and signal processing.

\section{Conclusion}

RF photonics has long been recognized as a key enabling technology for wideband antenna front-ends. RF photonic front-end processors have always provided bandwidth, but recent advances have shown dynamic range at high sensitivity as well. Continued advances in broadband link/processor performance as well as cost reduction are needed to maintain advantage over competing electronic front-end solutions. As photonics begin to creep into RF system architectures, more processing functionality can be expected. Until that time comes, RF photonics will remain a niche antenna technology along transmit and receive RF chains.

\section{References}

[1] H. Roussell, M. Regan, J. Prince, C. Cox, J. Chen, W. Burns, E. Ackerman, and J. Campbell, "Gain, noise figure, and bandwidth-limited dynamic range of a low-biased external modulation link," in Proc. IEEE Int. Topical Meeting Microw. Photon., p. 84, 2007.

[2] T. R. Clark, S. R. O'Connor, and M. L. Dennis, "A Phase-Modulation I/Q-Demodulation Microwave-to-Digital Photonic Link," IEEE Trans. Microw Theory Tech., Vol. 58, No. 11, p. 3039, 2010.

[3] X. Yao and L. Maleki, “High frequency optical subcarrier generator,” Electron. Lett., Vol. 30, p. $1525,1994$.

[4] A. Savchenkov, V. IIchenko, A. Matsko and L. Maleki, "Kilohertz optical resonances in dielectric crystal cavities," Phys. Rev. A, Vol. 70, p. 051804, 2004.

[5] A. Savchenkov, V. Ilchenko, A. Matsko, and L. Maleki, "High-Order Tunable Filters Based on a Chain of Coupled Crystalline Whispering Gallery-Mode Resonators," IEEE Photon. Technol. Lett., Vol. 17, No. 1, p. $136,2005$.

[6] C-S. Brès, S. Zlatanovic, A. O. J. Wiberg, J.R. Adleman, C.K. Huynh, E. W. Jacobs, J. M. Kvavle, and S. Radic, "Parametric Photonic Channelized RF Receiver," IEEE Photon. Technol. Lett., Vol. 23, No. 6, p. $344,2011$.

[7] S. Gupta and B. Jalali, "Time-warp correction and calibration in photonic time-stretch analog-to-digital converter," Opt. Lett., Vol. 33, Issue 22, p. 2674, 2008. 